\title{
GEOMETRIC PROPERTIES OF COMPOSITION OPERATORS BELONGING TO SCHATTEN CLASSES
}

\author{
YONGSHENG ZHU
}

(Received 9 August 1999)

\begin{abstract}
We investigate the connection between the geometry of the image domain of an analytic function mapping the unit disk into itself and the membership of the composition operator induced by this function in the Schatten classes. The purpose is to provide solutions to Lotto's conjectures and show a new compact composition operator which is not in any of the Schatten classes.
\end{abstract}

2000 Mathematics Subject Classification. 47B10, 47B33, 47B38, 30H05, 46E22.

1. Introduction. Let $D$ denote the unit disk in the complex plane $\mathbb{C}$ and let $H^{p}$ denote the Hardy space of functions

$$
f(z)=\sum_{n=0}^{\infty} a_{n} z^{n}
$$

analytic in $D$ such that

$$
\|f\|_{p}^{p}=\sup _{0 \leq r<1} \frac{1}{2 \pi} \int_{0}^{2 \pi}\left|f\left(r e^{i \theta}\right)\right|^{p} d \theta<\infty,
$$

where $0<p<\infty$. Let $\phi$ be an analytic function mapping $D$ into itself. The composition operator $C_{\phi}$ (induced by $\phi$ ) on $H^{p}$ is defined by

$$
C_{\phi}(f)(z)=f(\phi(z)), \quad z \in D .
$$

It is well known that $C_{\phi}$ is a bounded linear operator on $H^{p}$. The compactness of this operator is characterized in Shapiro [4] by the following criterion.

SHAPIRO'S COMPACTNESS CRITERION. The operator $C_{\phi}$ is compact if and only if

$$
\lim _{|z| \rightarrow 1} \frac{N_{\phi}(z)}{\log (1 /|z|)}=0
$$

where

$$
N_{\phi}(w)=\sum_{z \in \phi^{-1}(w)} \log \frac{1}{|z|}, \quad w \in D-\{\phi(0)\} .
$$

Let $d \lambda=\left(1-|z|^{2}\right)^{-2} d A$ be the Mobius invariant measure on $D$. Let $S_{p}\left(H^{2}\right)$ be the Schatten ideal of operators on the Hilbert space $H^{2}$ for $p>0$. D. H. Luecking and K. Zhu [3] proved the following theorem. 


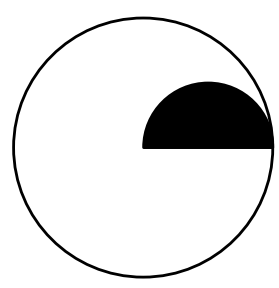

FIGURE 1.1

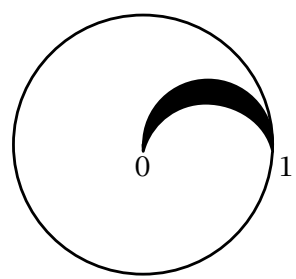

FIGURE 1.2

LUECKING-ZHU THEOREM. The composition operator $C_{\phi} \in S_{p}\left(H^{2}\right)$ if and only if

$$
\frac{N_{\phi}(z)}{\log (1 /|z|)} \in L^{p / 2}(d \lambda)
$$

B. A. Lotto [2] began the investigation of the connection between the geometry of $\phi(D)$ and the membership of composition operators in the Schatten classes.

Let

$$
\phi(z)=\frac{1}{1-i g(z)}, \quad \text { where } g(z)=\sqrt{i \frac{1-z}{1+z}} .
$$

Then $\phi$ is a Riemann map from $D$ onto the semi-disk

$$
\left\{z: \operatorname{Im}(z)>0 \text { and }\left|z-\frac{1}{2}\right|<\frac{1}{2}\right\}
$$

with $\phi(1)=1$ (see Figure 1.1).

Lotto proved that $C_{\phi}$ is a compact operator but not a Hilbert-Schmidt (i.e., $S_{2}\left(H^{2}\right)$ ) operator. His investigation led to the following conjecture.

LOTTO'S CONJECTURE 1. The composition operator $C_{\phi}$ belongs to the Schatten ideal $S_{p}$, if $p>2$.

Suppose $\psi$ is a univalent map from $D$ onto a crescent shaped region bounded by the semi-circle

$$
\left\{z: \operatorname{Im}(z) \geq 0 \text { and }\left|z-\frac{1}{2}\right|=\frac{1}{2}\right\}
$$

and a circular arc in the upper half of $D$ that joins 0 to 1 (see Figure 1.2).

Lotto proved that $C_{\psi}$ is a Hilbert-Schmidt operator and issued the following challenge. 
LOTTO'S CONJECTURE 2. Given $p, 0<p<\infty$, there exists a simple example of a domain $G_{p}$ with $G_{p} \subset D$, or there are easily verifiable geometric conditions on $G_{p}$, such that the Riemann map from $D$ onto $G_{p}$ induces a compact operator that is not in $S_{p}$.

He described a way to produce a compact composition operator which is not in any Schatten ideal if his conjecture 2 is true. Here we want to point out that Tom Carrol and Carl C. Cowen gave such an example in [1]. But the function $\phi$ that induces the desired compact non-Schatten class operator is described in terms of its "model for iteration." In general, it is hard to visualize the domain $\phi(D)$ and how the geometry of this domain prevents $C_{\phi}$ to belong to $S_{p}$ for all $p, 0<p<\infty$.

The goal of this paper is to prove both Lotto's conjectures. We establish Lotto's conjecture 1 in Section 3 and Lotto's conjecture 2 in Section 4. In Section 5, we follow Lotto's method to construct a Riemann map that induces a compact composition operator which is not in any Schatten ideals.

2. Background and terminology. For infinite-dimensional Hilbert space $H$ and compact operator $T$ on $H$, we define singular numbers for $T$ by

$$
s_{n}=s_{n}(T)=\inf \{\|T-K\|: K \text { has rank } \leq n\} .
$$

We know that the compact operators are exactly those $T$ for which $s_{n}(T) \rightarrow 0$. By definition the finite rank operators are those for which $s_{n}$ is eventually zero. In between are the Schatten classes. Specifically, the Schatten $p$-class $S_{p}(H), 0<p<\infty$, consists of those $T$ for which

$$
\sum_{n=0}^{\infty} s_{n}^{p}<\infty .
$$

The class $S_{1}(H)$ is the trace class and $S_{2}(H)$ is the famous Hilbert-Schmidt class. Clearly, $S_{p}(H) \subset S_{q}(H)$, if $0<p<q<\infty$.

We denote the set of bounded operators on $H$ by $B(H)$ and the set of compact operators on $H$ by $K(H)$. We have the following lemma.

LEMMA 2.1 (see [3]). The Schatten class $S_{p}(H), 0<p<\infty$, is a two-sided ideal in $B(H)$.

Let $\Omega_{n}(n=0,1,2)$ be simply connected domains such that $\Omega_{0} \subset \Omega_{1} \subset \Omega_{2} \subset D$ and $\rho_{n}$ be univalent maps from $D$ onto $\Omega_{n}$, respectively. The following useful corollaries are easy consequences of this lemma (see [2]).

COROLlaRY 2.2. If $C_{\rho_{1}} \in S_{p}(H)$ for some $p, 0<p<\infty$, then $C_{\rho_{0}} \in S_{p}(H)$.

COROLlaRY 2.3. If $C_{\rho_{1}} \notin S_{p}(H)$ for some $p, 0<p<\infty$, then $C_{\rho_{2}} \notin S_{p}(H)$.

COROLLARY 2.4. Suppose that $\Omega$ is the image of $\Omega_{1}$ under an automorphism of the unit disk $D$ and $\rho$ is a univalent analytic function which maps $D$ onto $\Omega$. Then $C_{\rho_{1}} \in$ $S_{p}(H)$ if and only if $C_{\rho} \in S_{p}(H)$.

If $\phi$ is univalent, we have

$$
N_{\phi}(w)=\log \frac{1}{\left|\phi^{-1}(w)\right|}
$$


that is equivalent to $1-\left|\phi^{-1}(w)\right|$, as $\left|\phi^{-1}(w)\right| \rightarrow 1$. Thus, Shapiro's compactness criterion becomes the following corollary.

COROLLARY 2.5. Suppose that $\phi$ is a univalent selfmap of $D$. The composition operator $C_{\phi}$ is compact on $H^{2}$ if and only if

$$
\lim _{|z| \rightarrow 1} \frac{1-|\phi(z)|}{1-|z|}=\infty
$$

Luecking-Zhu theorem implies the following corollary.

COROLlary 2.6. Suppose that $\phi$ is a univalent selfmap of $D$ into itself. The composition operator $C_{\phi} \in S_{p}\left(H^{2}\right)$ if and only if

$$
\chi_{\phi(D)} \cdot \frac{1-\left|\phi^{-1}(z)\right|}{1-|z|} \in L^{p / 2}(d \lambda) .
$$

We use Corollaries 2.5 and 2.6 to prove our theorems.

\section{Proof of Lotto's conjecture 1}

THEOREM 3.1. Let $\phi$ be a Riemann map from $D$ onto the semi-disk

$$
G=\left\{z: \operatorname{Im}(z)>0 \text { and }\left|z-\frac{1}{2}\right|<\frac{1}{2}\right\},
$$

such that $\phi(1)=1$. Then the composition operator $C_{\phi}$ induced by $\phi$ belongs to Schatten ideals $S_{p}$ for all $p>2$.

Proof. Since $\partial G$ contacts $\partial D$ only at $z=1$, we only need to consider what happens when $z \in G$ closes to 1 . For small $\varepsilon>0$, let $\Delta(\varepsilon)=\{z:|z-1|<\varepsilon\}$. By Corollary 2.6, we need to prove

$$
\int_{G \cap \Delta(\varepsilon)}\left(\frac{1-\left|\phi^{-1}(z)\right|}{1-|z|}\right)^{p / 2} \frac{d A(z)}{\left(1-|z|^{2}\right)^{2}}<\infty, \text { if } p>2 .
$$

It is not difficult to find such $\phi$ so that $\phi^{-1}(z)=\left(z^{2}-(z-1)^{2} i\right) /\left(z^{2}+(z-1)^{2} i\right)$ and

$$
1-\left|\phi^{-1}(z)\right|^{2}=-\frac{4 \operatorname{Im}(\bar{z}(z-1))^{2}}{\left|z^{2}+(z-1)^{2} i\right|} .
$$

Let $z=a+b i \in G$, then $\operatorname{Im}(\bar{z}(z-1))^{2} \approx 2 a b(a-1) \approx \operatorname{Im}(z-1)^{2}$, as $z \rightarrow 1$. Where $\approx$ means comparable. $A(t)$ and $B(t)$ are comparable if there are positive constants $C_{1}$ and $C_{2}$ such that $C_{1} A(t) \leq B(t) \leq C_{2} A(t)$. So we have

$$
1-\left|\phi^{-1}(z)\right|^{2} \approx-\operatorname{Im}(z-1)^{2}, \quad \text { as } z \longrightarrow 1, z \in G .
$$

Let $z=1-r e^{-i \theta} \in G$. Then $1-z=r e^{-i \theta} \in G$ which implies that $r^{2} \leq r \cos \theta$. Thus

$$
1-|z| \approx 1-|z|^{2}=r^{2}+2 r \cos \theta \approx r \cos \theta .
$$




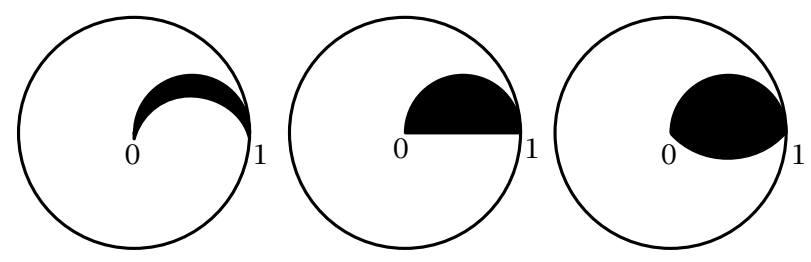

FIGURE 4.1

Now we use (3.4) and (3.5) to show that (3.2) is true. In fact,

$$
\begin{aligned}
\int_{G \cap \Delta(\varepsilon)}\left(\frac{1-\left|\phi^{-1}(z)\right|}{1-|z|}\right)^{p / 2} \frac{d A(z)}{\left(1-|z|^{2}\right)^{2}} & \\
& \approx \int_{G \cap \Delta(\varepsilon)}\left(\frac{1-\left|\phi^{-1}(z)\right|^{2}}{1-|z|}\right)^{p / 2} \frac{d A(z)}{(1-|z|)^{2}} \\
& \approx \int_{G \cap \Delta(\varepsilon)}\left(\frac{-\operatorname{Im}(z-1)^{2}}{1-|z|}\right)^{p / 2} \frac{d A(z)}{(1-|z|)^{2}} \\
& \approx \int_{G \cap \Delta(\varepsilon)}\left(\frac{r^{2} \sin 2 \theta}{r \cos \theta}\right)^{p / 2} \frac{r d r d \theta}{(r \cos \theta)^{2}}, \quad z=1-r e^{-i \theta}
\end{aligned}
$$

The last integral is finite if and only if the following integral:

$$
\int_{G}\left(\frac{r^{2} \sin 2 \theta}{r \cos \theta}\right)^{p / 2} \frac{r d r d \theta}{(r \cos \theta)^{2}}
$$

is finite. But

$$
\begin{aligned}
\int_{G}\left(\frac{r^{2} \sin 2 \theta}{r \cos \theta}\right)^{p / 2} \frac{r d r d \theta}{(r \cos \theta)^{2}} & =2^{p / 2} \int_{0}^{\pi / 2} \int_{0}^{\cos \theta}(r \sin \theta)^{p / 2} \frac{d r d \theta}{r \cos ^{2} \theta} \\
& \approx \int_{0}^{\pi / 2} \frac{d \theta}{(\pi / 2-\theta)^{2-p / 2}}<\infty, \text { if and only if } p>2
\end{aligned}
$$

This completes the proof of Theorem 3.1.

4. Geometric characterization of Schatten ideals. For $0<\alpha<1$, let $G_{\alpha}$ represents one of the shaded regions in Figure 4.1.

Each of the regions is bounded by the semi-circle

$$
\left\{z: \operatorname{Im}(z) \geq 0 \text { and }\left|z-\frac{1}{2}\right|=\frac{1}{2}\right\}
$$

and a circular arc that is inside of $D$ joining 0 to 1 (see Figure 4.1). These two arcs form angles of $\alpha \pi$ at 0 and 1. Define $\phi_{\alpha}$ to be one of the Riemann maps from $D$ onto $G_{\alpha}$ such that $\phi_{\alpha}(1)=1$. 

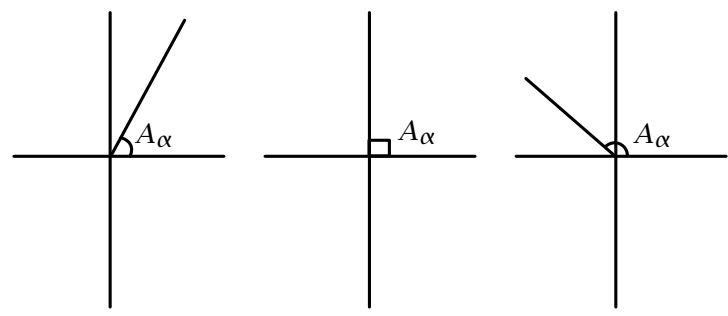

FIGURE 4.2

THEOREM 4.1. Suppose that $C_{\phi_{\alpha}}$ is the composition operator induced by $\phi_{\alpha}$. Then

(a) $C_{\phi_{\alpha}}$ does not belong to Schatten ideal $S_{2 \alpha /(1-\alpha)}\left(H^{2}\right)$.

(b) $C_{\phi_{\alpha}} \in S_{p}\left(H^{2}\right)$ for any $p>2 \alpha /(1-\alpha)$.

Theorem 4.1 gives Lotto's conjecture 2 a positive answer: given any $0<p<\infty$, we can pick $\alpha=p /(p+2) \in(0,1)$, then $G_{\alpha} \subset D$ is the domain for which the Riemann map from $D$ onto $G_{\alpha}$ induces a composition operator that is not in $S_{p}\left(H^{2}\right)$.

LEMMA 4.2. $\operatorname{Let} \tau(z)=i(1 / z-1)$. Then $\tau$ maps $G_{\alpha}$ onto the sector $A_{\alpha}$ (see Figure 4.2), where the two sides of the sector form an angle of $\alpha \pi$. Moreover,

$$
1-|z|^{2} \approx \rho^{2}+2 \rho \sin \theta, \quad \text { if } \tau(z)=\rho e^{i \theta}, \rho \longrightarrow 0 .
$$

Proof. It is easy to verify that $\tau\left(G_{\alpha}\right)=A_{\alpha}$ and

$$
\begin{aligned}
1-|z|^{2} & =1-\left|\frac{1}{1-i \tau}\right|^{2} \\
& \approx \rho^{2}+2 \rho \sin \theta, \quad \text { if } \tau(z)=\rho e^{i \theta}, \rho \longrightarrow 0 .
\end{aligned}
$$

LEMMA 4.3. Suppose that $w(\sigma)=(1+i \sigma) /(1-i \sigma)$, then $w$ maps the upper-half plane $\mathrm{H}^{+}$onto $\mathrm{D}$ and

$$
1-|w| \approx \operatorname{Im} \sigma, \text { as } \sigma \longrightarrow 0(\text { or } w \longrightarrow 1), \sigma \in H^{+} .
$$

Proof. Let $w=r e^{i \theta}$. Then

$$
\sigma=i \frac{1-r e^{i \theta}}{1+r e^{i \theta}} \approx \frac{1}{2} \theta+\frac{1}{2}(1-r) i, \quad \text { as } w \rightarrow 1 .
$$

Therefore,

$$
\operatorname{Im} \sigma \approx 1-r
$$

On the other hand, we have

$$
1-|w|^{2} \approx-i(\theta+(1-r) i)+i(\theta-(1-r) i)=2(1-r) \approx \operatorname{Im} \sigma .
$$

Now we can begin to prove Theorem 4.1.

Proof of Theorem 4.1. Let $\psi_{\alpha}=\phi_{\alpha}^{-1}$. We can decompose $\psi_{\alpha}$

$$
w=\psi_{\alpha}(z)=w\left(\tau^{1 / \alpha}(z)\right) .
$$


By Corollary 2.6, we need to show that

$$
\int_{G_{\alpha}}\left(\frac{1-\left|\psi_{\alpha}(z)\right|}{1-|z|}\right)^{p / 2} \frac{d A(z)}{\left(1-|z|^{2}\right)^{2}}
$$

is finite for $p>2 \alpha /(1-\alpha)$. Actually we only need to consider what happens to (4.9) when $z$ closes to 1 . So we consider

$$
\begin{aligned}
\int_{G_{\alpha} \cap \Delta(\varepsilon)}\left(\frac{1-\left|\psi_{\alpha}(z)\right|}{1-|z|}\right)^{p / 2} \frac{d A(z)}{\left(1-|z|^{2}\right)^{2}} \\
\quad \approx \int_{G_{\alpha} \cap \Delta(\varepsilon)}\left(\frac{1-|w(z)|}{1-|z|}\right)^{p / 2} \frac{d A(z)}{(1-|z|)^{2}} \\
\approx \iint_{\tau\left(G_{\alpha} \cap \Delta(\varepsilon)\right)}\left(\frac{\operatorname{Im} \sigma}{\rho^{2}+2 \rho \sin \theta}\right)^{p / 2} \frac{\rho d \rho d \theta}{\left(\rho^{2}+2 \rho \sin \theta\right)^{2}},
\end{aligned}
$$

where $\sigma$ is in the upper-half plane, $(\rho, \theta) \in \tau\left(G_{\alpha} \cap \Delta(\varepsilon)\right) \subset A_{\alpha}$ and $\sigma=\tau^{1 / \alpha}$. Without loss of generality, we may assume that $\tau\left(G_{\alpha} \cap \Delta(\varepsilon)\right)=\left\{(\rho, \theta) \in A_{\alpha}: \rho<1\right\}$. Now we consider three cases: $0<\alpha<1 / 2 ; \alpha=1 / 2$; and $1 / 2<\alpha<1$.

CASE $1(0<\alpha<1 / 2)$. Define

$$
\begin{aligned}
& \Omega_{1}=\left\{(\rho, \theta) \in \tau\left(G_{\alpha} \cap \Delta(\varepsilon)\right): 0<\rho \leq 2 \sin \theta\right\} \\
& \Omega_{2}=\left\{(\rho, \theta) \in \tau\left(G_{\alpha} \cap \Delta(\varepsilon)\right): \rho>2 \sin \theta\right\} \\
& \int_{G_{\alpha}}\left(\frac{1-\left|\psi_{\alpha}(z)\right|}{1-|z|}\right)^{p / 2} \frac{d A(z)}{\left(1-|z|^{2}\right)^{2}} \\
& \approx \int_{\Omega_{1}}\left(\frac{\operatorname{Im} \sigma}{\rho^{2}+2 \rho \sin \theta}\right)^{p / 2} \frac{\rho d \rho d \theta}{\left(\rho^{2}+2 \rho \sin \theta\right)^{2}} \\
& \quad+\iint_{\Omega_{2}}\left(\frac{\operatorname{Im} \sigma}{\rho^{2}+2 \rho \sin \theta}\right)^{p / 2} \frac{\rho d \rho d \theta}{\left(\rho^{2}+2 \rho \sin \theta\right)^{2}} \\
& \approx \iint_{\Omega_{1}}\left(\frac{\rho^{1 / \alpha} \sin (\theta / \alpha)}{\rho \sin \theta}\right)^{p / 2} \frac{\rho d \rho d \theta}{(\rho \sin \theta)^{2}} \\
& \quad+\iint_{\Omega_{2}}\left(\frac{\rho^{1 / \alpha} \sin (\theta / \alpha)}{\rho^{2}}\right)^{p / 2} \frac{\rho d \rho d \theta}{\left(\rho^{2}\right)^{2}} \\
& \approx \int_{0}^{\alpha \pi} d \theta \int_{0}^{2 \sin \theta}\left(\frac{\rho^{1 / \alpha} \sin (\theta / \alpha)}{\rho \sin \theta}\right)^{p / 2} \frac{\rho d \rho}{(\rho \sin \theta)^{2}} \\
& \quad+\int_{0}^{\alpha \pi} d \theta \int_{2 \sin \theta}^{1}\left(\frac{\rho^{1 / \alpha} \sin (\theta / \alpha)}{\rho^{2}}\right)^{p / 2} \frac{\rho d \rho d \theta}{\left(\rho^{2}\right)^{2}} \\
& \approx \int_{0}^{\alpha \pi} \frac{1}{\theta^{2-(1 / \alpha-1)(p / 2)} d \theta .}
\end{aligned}
$$

The last integral converges if and only if $2-(1 / \alpha-1)(p / 2)<1$, that is, $p>$ $2 \alpha /(1-\alpha)$. This simultaneously proves both parts (a) and (b) of Theorem 4.1 for $0<\alpha<1 / 2$. 
CASE $2(\alpha=1 / 2)$. That is what exactly Theorem 3.1 is about.

CASE $3(1 / 2<\alpha<1)$. Define

$$
\begin{aligned}
& \Omega_{1}=\left\{(\rho, \theta) \in \tau\left(G_{\alpha} \cap \Delta(\varepsilon)\right): 0<\rho \leq 2 \sin \theta \text { and } \theta<\frac{\pi}{4}\right\}, \\
& \Omega_{2}=\left\{(\rho, \theta) \in \tau\left(G_{\alpha} \cap \Delta(\varepsilon)\right): \rho>2 \sin \theta\right\}, \\
& \Omega_{3}=\left\{(\rho, \theta) \in \tau\left(G_{\alpha} \cap \Delta(\varepsilon)\right): 0<\rho \leq 2 \sin \theta \text { and } \theta \geq \frac{\pi}{4}\right\}, \\
& \int_{\Omega_{3}}\left(\frac{1-\left|\psi_{\alpha}(z)\right|}{1-|z|}\right)^{p / 2} \frac{d A(z)}{\left(1-|z|^{2}\right)^{2}} \approx \iint_{\Omega_{3}}\left(\frac{\operatorname{Im} \sigma}{\rho^{2}+2 \rho \sin \theta}\right)^{p / 2} \frac{\rho d \rho d \theta}{\left(\rho^{2}+2 \rho \sin \theta\right)^{2}} \\
& \approx \int_{\pi / 4}^{\alpha \pi} \frac{1}{\theta^{2}} d \theta \int_{0}^{1} \rho^{(1 / \alpha-1)(p / 2-2)} d \rho .
\end{aligned}
$$

The last integral is finite if and only if $p>2 \alpha /(1-\alpha)$. Using the same proof as in Case 1, we have

$$
\begin{aligned}
& \int_{\Omega_{1}}\left(\frac{1-\left|\psi_{\alpha}(z)\right|}{1-|z|}\right)^{p / 2} \frac{d A(z)}{\left(1-|z|^{2}\right)^{2}}<\infty \text { if and only if } p>\frac{2 \alpha}{1-\alpha}, \\
& \int_{\Omega_{2}}\left(\frac{1-\left|\psi_{\alpha}(z)\right|}{1-|z|}\right)^{p / 2} \frac{d A(z)}{\left(1-|z|^{2}\right)^{2}}<\infty \text { if and only if } p>\frac{2 \alpha}{1-\alpha} .
\end{aligned}
$$

This completes the proof of Theorem 4.1.

Let

$$
T_{a, \theta}(z)=\frac{z+a}{1+a z} e^{i \theta}, \quad-1<a<1,0 \leq \theta<2 \pi .
$$

$T_{a, \theta}$ is an automorphism of $D$ and maps $G_{\alpha}$ onto $\Omega_{\alpha, a, \theta}$, where $\Omega_{\alpha, a, \theta}$ can have different shapes and positions, depending on the parameters. By choosing appropriate parameters, we can apply Theorems 3.1 and 4.1 and Corollary 2.4 to different regions. We can also apply these theorems with Corollaries 2.2 and 2.3. In Section 5, we use Theorem 4.1 and Corollary 2.4 to construct a non-Schatten class compact composition operator.

5. A non-Schatten class composition operator. Based on Lotto's suggestion, we successfully constructed a compact composition operator that is not in any Schatten ideals. Here is the process of the construction.

Let $\theta_{n}=\pi /(n+1), z_{n}=e^{i \theta_{n}}, r_{n}=(1 / 2) \sin \theta_{n}$, and $c_{n}=\left(1-r_{n}\right) z_{n}$, where $n=$ $1,2,3, \ldots$ Define $\Omega_{n}$ to be the region bounded by the semi-circle

$$
\left\{z: \operatorname{Im}(z) \geq 0 \text { and }|z-| c_{n}||=r_{n}\right\}
$$

and a circular arc that is inside $D$ joining $1-2 r_{n}$ to 1 (it is actually a line segment when $n=1)$. These two arcs form an angle of $(n+1) /(n+3) \pi$ at 1 . Let

$$
\begin{gathered}
\Omega_{n}^{\prime}=\left\{z e^{i \theta_{n}}: z \in \Omega_{n}\right\}, \\
\Omega=\cup_{n=1}^{\infty} \Omega_{n}^{\prime} .
\end{gathered}
$$

We have the following theorem. 


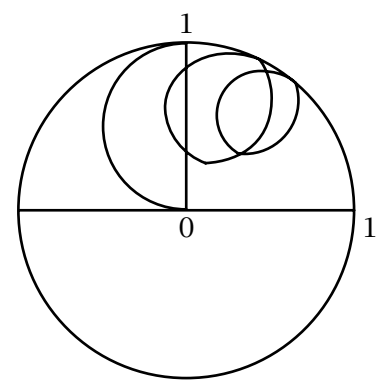

FIGURE 5.1

THEOREM 5.1. Suppose that $\Omega$ is defined by (5.3). Then we have

(1) $\Omega$ is a simply connected domain contained in the upper half of $D$.

(2) Any Riemann map $\phi$ that maps $D$ onto $\Omega$ induces a compact composition operator $C_{\phi}$. But $C_{\phi}$ does not belong to any Schatten ideal $S_{p}\left(H^{2}\right), p>0$.

Proof. We estimate the distance between the centers $c_{n-1}$ and $c_{n}$ of $\Omega_{n-1}^{\prime}$ and $\Omega_{n}^{\prime}(n \geq 2)$ :

$$
\begin{aligned}
\left|c_{n-1}-c_{n}\right| & =\left|\left(1-r_{n}\right) z_{n}-\left(\left(1-r_{n-1}\right) z_{n-1}\right)\right| \\
& =\left|\left(1-\frac{1}{2} \sin \frac{\pi}{n+1}\right) e^{i(\pi /(n+1))}-\left(\left(1-\frac{1}{2} \sin \frac{\pi}{n}\right) e^{i(\pi / n)}\right)\right| \\
& =O\left(\frac{1}{n^{2}}\right) .
\end{aligned}
$$

But the radius $r_{n}$ of $\Omega_{n}^{\prime}$ is $(1 / 2) \sin (\pi /(n+1)) \geq 1 /(1+n)$. Thus $\Omega_{n-1}^{\prime}$ and $\Omega_{n}^{\prime}$ overlap and $\Omega$ is simply connected.

Since

$$
\operatorname{Im} c_{n}=\left(1-r_{n}\right) \operatorname{Im} z_{n}=\left(1-r_{n}\right) \sin \frac{\pi}{n+1}>\frac{1}{2} \sin \frac{\pi}{n+1}=r_{n} .
$$

$\Omega_{n}^{\prime}$ lies in the upper half of $D$. Therefore $\Omega \subset D$ is in the upper plane. By the construction of $\Omega$, we know that $\Omega$ touches the boundary of $D$ only at $z_{n}, n=1,2,3, \ldots$, and 1 . One can see that at $z_{n}, \phi$ is not conformal (see $[4,5]$ ). Thus $\phi$ has no finite angular derivative at $z_{n}$. Note that $\Omega \subset D$ is in the upper plane and $z_{n} \rightarrow 1$ as $n \rightarrow \infty, \phi$ is not conformal at 1 either. By the angular derivative criterion for compactness (see [5]), we know $C_{\phi}$ is compact. Let $\phi_{n}$ be a Riemann map that maps $D$ onto $\Omega_{n}^{\prime}$ and $C_{n}$ be the induced composition operator. Let $G_{\alpha}$ be the region defined in Section 4 and $\psi_{\alpha}$ be a Riemann map from $D$ onto $G_{\alpha}$. By Theorem 4.1, we know that the composition operator induced by $\psi_{(n+1) /(n+3)}$ does not belong to Schatten ideal $S_{n+1}$. Let

$$
\eta_{n}(z)=\frac{z+\left(1-2 r_{n}\right)}{1+\left(1-2 r_{n}\right) z} e^{i \theta}, \quad z \in D
$$

Then $\eta_{n}$ is an automorphism of $D$. Moreover, $\eta_{n}\left(G_{(n+1) /(n+3)}\right)=\Omega_{n}^{\prime}$ (see Figure 5.2). Thus, according to Corollary 2.4, $C_{n} \notin S_{n+1}\left(H^{2}\right)$. But $\Omega_{n}^{\prime} \subset \Omega$ for any positive integer $n, C_{\phi} \notin S_{n+1}\left(H^{2}\right)$ by Corollary 2.3 for any $n$. We know that $S_{p}\left(H^{2}\right) \subset S_{q}\left(H^{2}\right)$ if $p<q$. Therefore $C_{\phi}$ does not belong to any Schatten classes. This completes the proof of Theorem 5.1. 


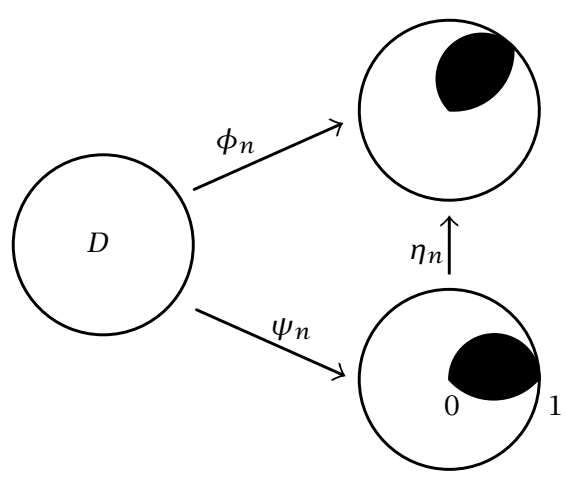

FIGURE 5.2

ACKNOWLEDGEMENTS. It is a great pleasure for me to express my sincere gratitude to my advisor, Dr David Stegenga, for his understanding, patient guidance, and valuable suggestions. Thanks also go to Dr Wayne Smith and Dr George Csordas.

\section{REFERENCES}

[1] T. Carroll and C. C. Cowen, Compact composition operators not in the Schatten classes, J. Operator Theory 26 (1991), no. 1, 109-120. MR 94c:47045. Zbl 792.47031.

[2] B. A. Lotto, A compact composition operator that is not Hilbert-Schmidt, Studies on Composition Operators, Contemporary Mathematics, vol. 213, Amer. Math. Soc., Rhode Island, 1998, pp. 93-97. MR 98j:47071. Zbl 898.47025.

[3] D. H. Luecking and K. H. Zhu, Composition operators belonging to the Schatten ideals, Amer. J. Math. 114 (1992), no. 5, 1127-1145. MR 93i:47032. Zbl 792.47032.

[4] J. H. Shapiro, The essential norm of a composition operator, Ann. of Math. (2) 125 (1987), no. 2, 375-404. MR 88c:47058. Zbl 642.47027.

[5]___ Composition Operators and Classical Function Theory, Universitext: Tracts in Mathematics, Springer-Verlag, New York, 1993. MR 94k:47049. Zbl 791.30033.

YongSheng Zhu: FAIR IsAaC 2150 ShatTuck AVEnue, Berkeley, CA 94704, USA

E-mail address: zhuys@hawai i . edu

URL: http://www2.hawaii.edu/ ${ }^{2}$ zhuys 


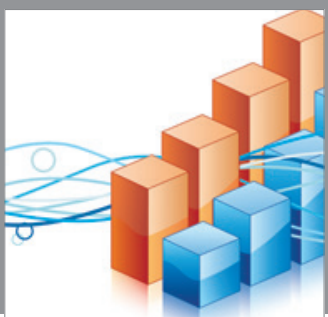

Advances in

Operations Research

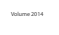

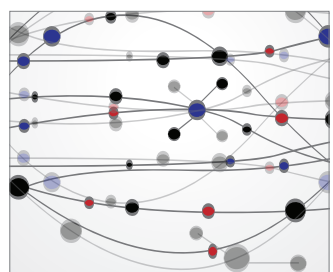

\section{The Scientific} World Journal
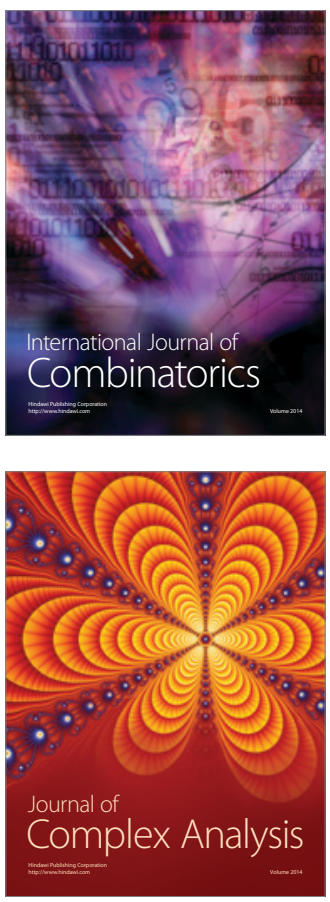

International Journal of

Mathematics and

Mathematical

Sciences
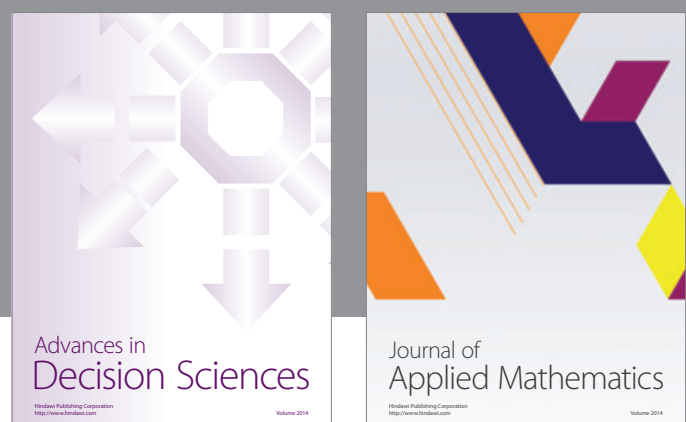

Journal of

Applied Mathematics
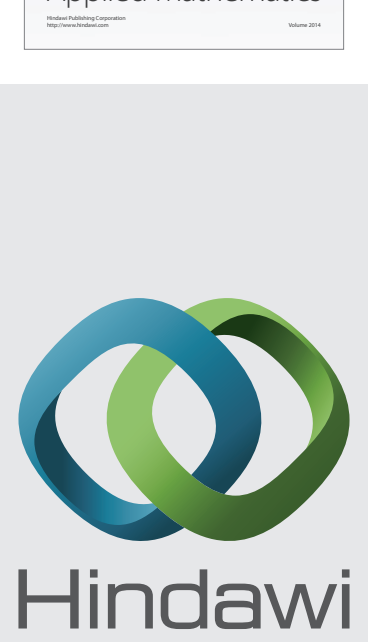

Submit your manuscripts at http://www.hindawi.com
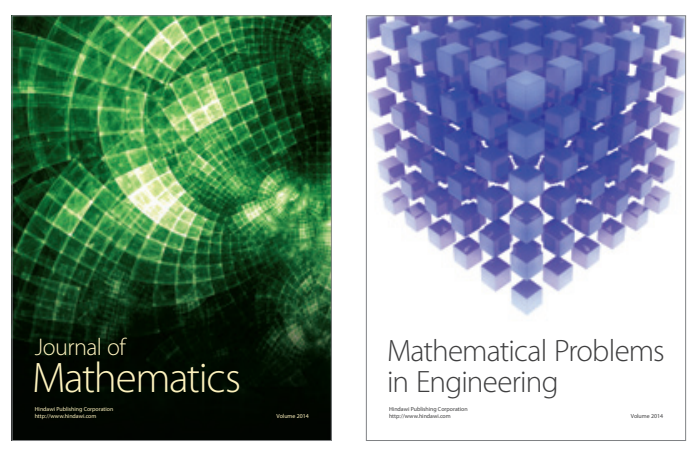

Mathematical Problems in Engineering
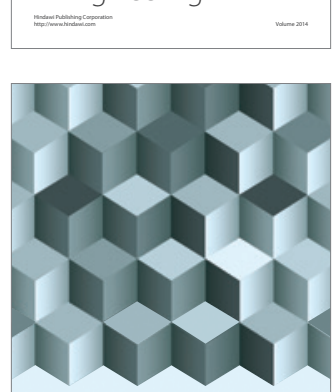

Journal of

Function Spaces
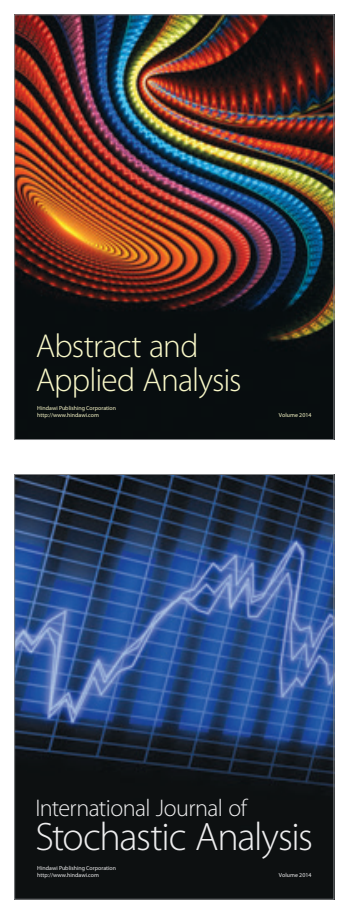

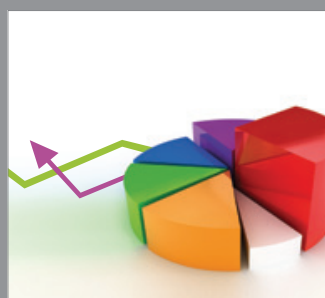

ournal of

Probability and Statistics

Promensencen
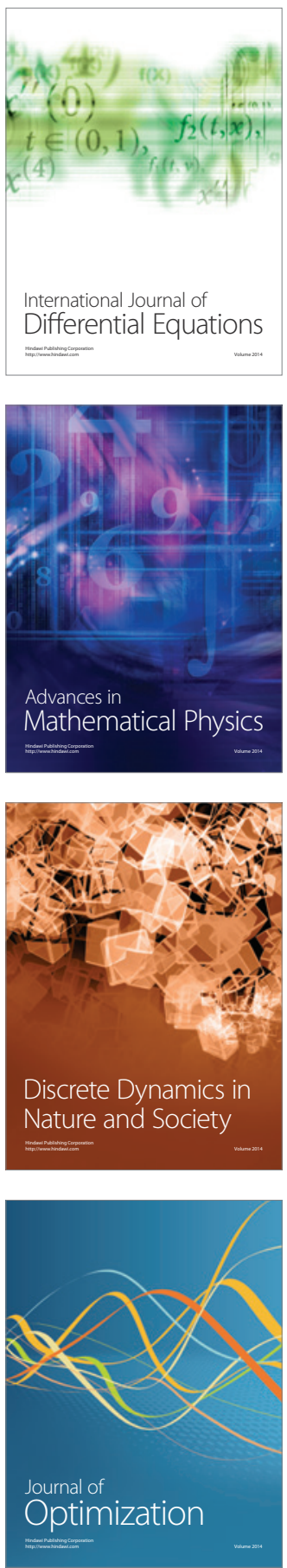